\title{
Miranda
}

Revue pluridisciplinaire du monde anglophone /

Multidisciplinary peer-reviewed journal on the English-

speaking world

$10 \mid 2014$

Images on the Move: Circulations and Transfers in film

\section{Chroniques de l'écran. Les apports de la proxémie dans l'élaboration d'une nouvelle taxinomie.}

\section{Cécile Martin}

\section{OpenEdition}

Journals

Édition électronique

URL : http://journals.openedition.org/miranda/8816

DOI : 10.4000/miranda.8816

ISSN : 2108-6559

Éditeur

Université Toulouse - Jean Jaurès

\section{Référence électronique}

Cécile Martin, « Chroniques de l'écran. Les apports de la proxémie dans l'élaboration d'une nouvelle taxinomie. », Miranda [En ligne], 10 | 2014, mis en ligne le 09 mars 2016, consulté le 16 février 2021. URL : http://journals.openedition.org/miranda/8816 ; DOI : https://doi.org/10.4000/miranda.8816

Ce document a été généré automatiquement le 16 février 2021.

\section{cc) (i) () $\Theta$}

Miranda is licensed under a Creative Commons Attribution-NonCommercial-NoDerivatives 4.0 International License. 


\title{
Chroniques de l'écran. Les apports de la proxémie dans l'élaboration d'une nouvelle taxinomie.
}

\author{
Cécile Martin
}

1 Depuis le milieu du XXème siècle, de nombreux dispositifs sont venus concurrencer l'écran de cinéma dans sa fonction de diffusion d'images animées. Toutefois, si la multiplication des écrans peut être le signe de la fin du monopole de l'écran de cinéma, ce phénomène ne peut-il aussi s'envisager comme une opportunité pour aborder l'ontologie de l'écran?

2 Pour déterminer ses spécificités, nous envisagerons dans un premier temps l'écran d'un point de vue historique. En replaçant l'écran de cinéma dans une perspective chronologique nous verrons comment il s'est démarqué d'autres dispositifs et quels aspects des modèles d'écran antérieurs y ont été conservés. Ces éléments seront un préalable à une discussion sur la validité des critères de classification des dispositifs écraniques, critères remis en cause par les mutations intervenues depuis la seconde moitié du XX⿳亠丷⿵冂丶 ${ }^{\text {èe }}$ siècle dans les modalités de circulation des images animées. Nous verrons notamment comment des notions qui sollicitent une approche spatialisante des dispositifs de visualisation, telles que l'interopérabilité et la mobilité, peuvent être des indices de classification pertinents pour appréhender les nouveaux écrans.

\section{L'écran avant le cinéma}

3 À la fin du XIIİ̀me siècle, l'écran est un " panneau servant à se garantir de l'ardeur d'un foyer » («Écran») qui fait partie du mobilier des grandes demeures de la Maison de France, des comtés de Flandres et de Hainaut et du duché de Brabant. Il est référencé dans le Dictionnaire du Moyen Français (1330-1500) sous plusieurs formes attestées telles que " écran, écrane, escramaille, escramare, escramelle, escramoire » ("Scherm») et prendra sa forme définitive écran au milieu du XVI ${ }^{\text {ème }}$ siècle. 
4 D'une facture plus utilitaire qu'esthétique, l'écran devient néanmoins le support où les artisans laissent s'exprimer leur créativité, avec plus ou moins de succès :

On dit c'est un ignorant, qui n'a jamais appris le Blason que dans les écrans. C'est un pauvre Poète qui ne fait des vers que pour les écrans (Furetière 985).

Les nombreux témoignages écrits et l'apparition d'emplois du terme au second degré permettent de se rendre compte de la popularité dont jouit ce meuble à partir du milieu du XVIII ${ }^{\mathrm{ème}}$ siècle :

On dit à celui qui se met devant un autre pour empêcher qu'il ne se chauffe, Otez vous, je ne veux point d'un écran si épais. On dit encore par antiphrase d'une porte ouverte, voilà un vilain écran (Furetière 985).

6 Portant un nouveau regard sur leurs écrans, les "écraniers" s'intéressent non seulement aux aspects décoratifs, mais surtout à son potentiel en matière de divertissement, et rivalisent d'inventivité, mettant en jeu différentes techniques broderie, sculpture, peinture -, interrogeant ses propriétés mécaniques à des fins esthétiques, comme nous pouvons le voir dans cet exemple daté du premier tiers du XIX ̀̀me siècle :

On fait depuis quelques temps, des petits écrans très jolis et très curieux. Les uns se montrent un paysage d'hiver, les arbres dépouillés de feuilles ; mais en les approchant du feu, les feuilles, les fleurs, les fruits même se montrent, et la nature parait dans son printemps. Cet effet est facile à obtenir; on peint les arbres et le fond du paysage avec des couleurs invariables, les feuilles et les fruits avec des encres de sympathie, qui ne paraissent que lorsqu'elles ont reçu un certain degré de chaleur, et qui disparaissent au fur et à mesure que l'écran se refroidit (Francoeur 279).

7 Si la filiation avec l'écran de cheminée et la proximité de la chaleur sont encore sensibles dans certains artefacts, ce sont surtout les propriétés de la lumière et les jeux de transparence qui seront peu à peu mobilisés :

D'autres [écrans] présentent, sur un papier transparent, un sujet quelconque, lorsqu'on le regarde par-dessus et au jour ou à la lumière ; mais si l'on cache le jour ou la lumière avec l'écran, le sujet qu'on a regardé ne s'aperçoit plus, on en voit un autre tout différent qui parait en transparent (Francoeur 279).

8 L'aspect narratif tient également une part importante dans de nouveaux dispositifs popularisés depuis la fin du XVIII ${ }^{2}$ siècle par Louis de Carmontelle. Parmi ces derniers, citons le cas de l'écran-panorama :

M. Gaucheret, de Paris, prit en 1820 un brevet d'invention de cinq ans, pour de jolis écrans qu'il nomme écrans-panoramas. Des scènes disposées sur des bandes à peu près de deux aunes (deuxx mètres vingt centimètres) de long, se succèdent au moyen d'un mécanisme très simple, et offrent aux yeux les changements des saisons, les dangers des tempêtes, les divers personnages d'une mascarade, en ombres chinoises (Francoeur 279).

Dès le XIX ${ }^{\text {ème }}$ siècle on peut donc distinguer différents types d'écrans, avec d'une part les éléments de mobilier :

les écrans à pivot, fixes, à coulisses, à balustres, à médaillons, à bannière [...] se posant devant le feu ou s'accrochant au manteau de la cheminée [et] les écrans qu'on tenait à la main pour se garantir le visage (Havard 321).

Qu'ils soient brodés, sculptés ou peints, ces éléments ne présentent qu'une image fixe. On les distinguera alors des artefacts dans lesquels plusieurs images se succèdent sur un même support, annonçant l'ère de l'écran comme dispositif de médiation des images qui débute à la fin du XIX ${ }^{\text {ème }}$ siècle avec l'invention du cinématographe. 


\section{Cartographie}

11 Pour analyser les dispositifs écraniques précédant l'invention du cinématographe, nous avons réalisé un diagramme en vue d'établir une taxinomie. Pour sélectionner les critères présidant à l'ordonnancement des objets étudiés, nous nous sommes basés sur deux aspects notables qui ont permis de distinguer le dispositif cinématographique parmi d'autres inventions contemporaines, à savoir le caractère "collectif » et " public » de la projection. ${ }^{1}$

Nous avons placé ces notions sur deux axes, l'un opposant collectif à individuel, l'autre public à privé, pour réaliser un premier graphique où sont représentés les dispositifs que nous avons décrits précédemment ainsi qu'un certain nombre de jouets d'optique.

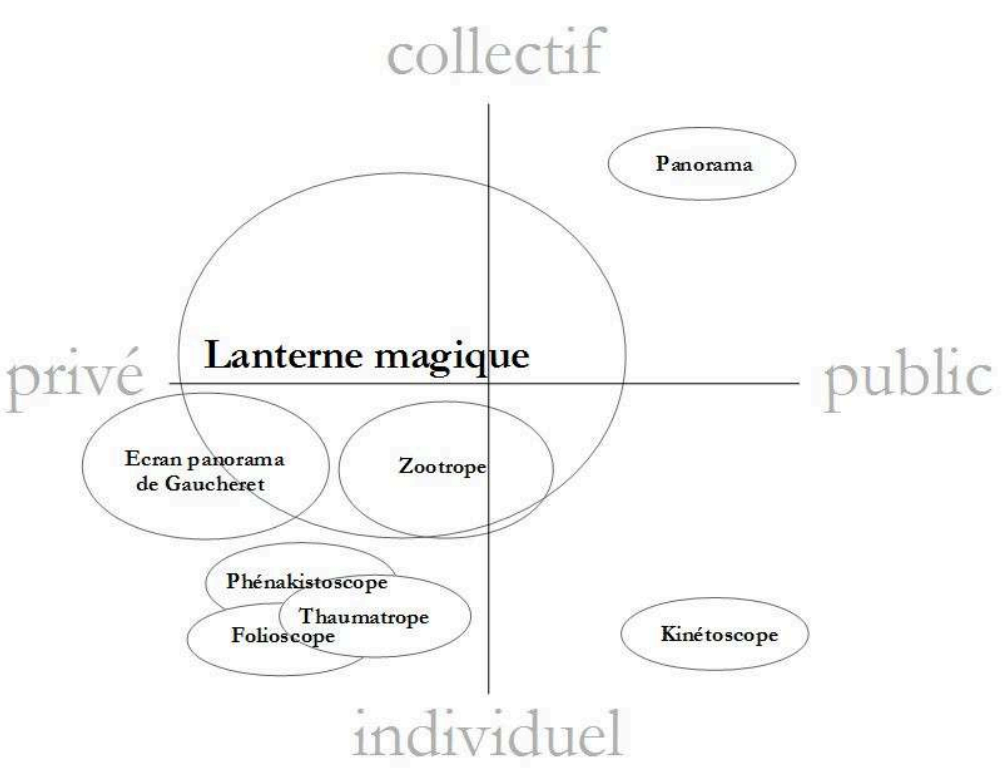

Ce choix se justifie de plusieurs façons. Certes ils ne comportent pas d'écran de projection, et c'est d'ailleurs pour ce motif qu'il leur est parfois refusé une place dans l'histoire du cinéma par les chercheurs qui considèrent que c'est «la projection [qui] fonde le cinéma » (Désile 45). Mais ces dispositifs proposent ce qu'on appellera un effet d'écran, au sens où ils ne comportent pas de surface sur laquelle viendraient s'actualiser des images éphémères, mais où il y a bien succession d'images dans un même espace.

C'est en tout cas l'expérience offerte par le Folioscope, aussi appelé Feuilletoscope, Flipbook ou encore Feuilleteur :

Cahier inventé en 1769 par l'artiste allemand Philippe Jacob Lautenberg, dont chaque page comporte le dessin d'une figure [...]. Les mouvements de la figure sont décalés sur chacune des pages; en faisant tourner les pages, on voit la figure en mouvement (Roy 174).

L'impression de mouvement est obtenue par le défilement des images dans la même portion du champ visuel de l'usager, procédé utilisé également par le Phénakistiscope : 
Jouet mis au point en 1832 par le Belge Joseph-Antoine Plateau créant l'illusion du mouvement. Le Phénakistiscope est composé d'un cercle troué de petites meurtrières à l'intérieur desquelles se trouvent des figures, [et qui], appliqué sur l'œil et tournant sur lui-même [...] transforme les images en une suite continue de mouvements perçue grâce à la persistance rétinienne (Roy 342).

Il est intéressant de noter que ces deux dispositifs ne permettent qu'à un nombre limité de spectateurs de profiter du spectacle simultanément. En effet la portion d'espace où se situe la figure animée est d'une taille relativement restreinte. Ces dispostifis ne peuvent donc faire l'objet d'une exploitation collective.

17 Cette contrainte apparaît de manière évidente dans le cas du Thaumatrope :

Jouet inventé dans les années 1820 [...] La rotation rapide d'un disque en carton, tendu entre deux fils et dont chacune des faces comporte un dessin différent, provoque la superposition visuelle des deux dessins (l'oiseau d'un côté et la cage de l'autre) (Roy 437).

Si l'on ajoute à cela la brièveté de l'animation qui ne permet pas d'envisager l'organisation de séances, ces différents aspects restreignent donc le "pré-cinéma » (Aumont et Marie 164) à des usages individuels et privés. Les améliorations proposées pour ces dispositifs viseront donc à dépasser ces contraintes, comme on peut le voir dans le cas du Zootrope ou Zoetrope (1834):

En tournant un tambour ouvert à sa partie supérieure et percé de petites fenêtres, le spectateur peut voir s'animer des personnages dessinés sur une bande de papier (Roy 473).

Celui-ci peut se concevoir comme une version améliorée du Phénakistiscope, puisqu'il est accessible à un plus grand nombre de spectateurs simultanément et allonge la durée de l'animation.

Concernant maintenant les dispositifs directement issus de la tradition historique de l'écran, on peut noter que les écrans à encre de sympathie et à effet de transparence pouvaient donner lieu à des démonstrations aussi bien individuelles que collectives, le principe de base autorisant des déclinaisons pour des écrans à main comme pour des écrans sur pied. Toutefois la fragilité de ces objets et, là encore, la durée de l'animation, était un frein à une exploitation publique.

21 C'est cet aspect qui différencia les transparents de Carmontelle (fin du XVIII ${ }^{\text {ème }}$ siècle) et l'écran-panorama de Gaucheret (1820). S'ils se prêtaient à des usages aussi bien individuels que collectifs, la durée de l'expérience, allongée par le principe du déroulement mécanique de la bande de papier, ouvrait la voie à une exploitation de l'écran à des fins non plus seulement démonstratives mais réellement narratives.

Ces exemples nous amènent au cas de la lanterne magique :

Appareil projetant des images agrandies, peintes sur verre et placées sur une lanterne [...] la lanterne magique est une chambre noire inversée. Elle est formée de trois éléments : une source lumineuse, une plaque de verre peinte et une lentille convergente [...] La première lanterne magique apparaît au XVII ${ }^{\mathrm{eme}}$ siècle et son usage se répand rapidement [...] Au fil des siècles on la modifie et l'améliore : ajout d'un miroir concave et d'autres lentilles pour condenser la lumière; source lumineuse de diverses natures (bougie, lampe à huile, ampoule); lanterne à double objectif permettant le fondu enchainé entre deux plaque de verre pour deux images. [Elle] sera très en vogue au milieu du XIX ${ }^{\text {ème }}$ siècle. (Roy 269-270). qu'elle sera perfectionnée au XIX ${ }^{\text {ème }}$ siècle pour répondre à deux objectifs : accroître 
l'audience d'une séance (travail sur la luminosité des images, augmentation de leur taille) et développer l'aspect narratif (enchaînement des images par la technique du fondu). spectacles collectifs et publics dont la durée ne soit pas conditionnée par la surface du support d'actualisation des images. En plus d'être l'élément visible du dispositif pour le spectateur, il contribue donc d'un point de vue technique au succès du cinéma, et très rapidement s'instaure un rapport métonymique entre les deux. Soutenu par de nouvelles expressions telles que "porter à l'écran» ou " adapter pour l'écran » ("Écran»), ce lien métaphorique tend à évincer la filiation de l'écran avec les dispositifs antérieurs. 
31 Apportant leur contribution à ce processus, de nombreux titres de revues et d'ouvrages fleurissent ${ }^{2}$ et confirment le nouveau sens du terme écran.

Cette littérature participe à populariser l'écran de projection, devenu l'écran de cinéma, voire simplement « l'écran », au détriment d'autres inventions contemporaines comportant des écrans :

S'appuyant sur les travaux du Britannique William Crookes (1832-1919), qui inventa le tube à rayons cathodiques, le physicien allemand Karl Ferdinand Braun (1850-1918) conçoit en 1897 un montage qui lui permet de rendre visible la forme d'un signal électrique. Dans un tube à vide, Braun dispose une électrode (la cathode) qui émet un faisceau d'électrons en direction d'un écran (relié à l'anode) recouvert d'une substance phosphorescente qui s'illumine à l'endroit de l'impact. Sur leur trajet, les électrons peuvent être déviés par une bobine qui les attire ou les repousse plus ou moins, selon le courant électrique qui la parcourt: le point d'impact lumineux sur l'écran se déplace ainsi le long d'un segment, au rythme du courant dans la bobine. ("Oscilloscope cathodique »).

Comme on peut le voir, l'écran occupe une place centrale dans l'oscilloscope cathodique de Braun, cependant il ne sera pas mis en avant pendant plusieurs décennies, et ce pour deux raisons. D'abord les usages de l'oscilloscope restent professionnels, ce dernier n'est donc pas mis à disposition du grand public. Ensuite on désignera les montages qui utilisent ce système d'affichage par des noms génériques qui ne mettent pas en avant spécifiquement l'écran, mais l'ensemble du dispositif, comme dans le cas du « radar». Ceci peut s'expliquer par le fait qu'à la différence du cinéma, où le projecteur est situé hors du champ de vision, laissant l'usager face au seul écran, ce même usager a ici à faire à des objets qu'il peut saisir ou embrasser du regard dans leur totalité.

Il faudra attendre la seconde moitié du XX ${ }^{\text {ème }}$ siècle pour que l'hégémonie de l'écran de cinéma commence à être remise en question. À partir des années 1950 on commencera à distinguer « le grand écran, pour le cinéma, et le petit écran, pour la télévision » (Roy 150).

Autre déclinaison de l'invention de Braun, les «écrans d'ordinateur " (Chantepie et Le Diberder 18) viendront à partir des années 1970 compléter l'offre de dispositifs de visualisation d'images animées. Bien qu'assez proches, « les écrans de nos téléviseurs et [les écrans] de nos ordinateurs " (De la Cotardière 169-170) qui ont pour origine commune les travaux sur les tubes de Crookes, vont mener une existence autonome jusqu'à la fin des années 1990.

36 La démocratisation de l'informatique, suivie par le développement des PDA (Personal Digital Assistant), pagers et téléphones mobiles, contribuera à la redéfinition du rapport métaphorique entre écran et cinéma, mais aussi à rendre floue la définition de l'écran. Ainsi, depuis le début du XXI ${ }^{\text {ème }}$ siècle, on a pu observer que l'écran de cinéma commence à se dissoudre dans des locutions telles que "l'écran transversal ", " ambiant ", ou «immersif» (Frau-Meigs 71-72), ou encore " l'écranosphère » ou « l'écran global» (Lipovetsky et Serroy 11)3. L'écran de cinéma fait donc partie d'un ensemble associant de nombreux dispositifs diffusant des images animées, association que nous allons à présent tenter de clarifier.

Si ce phénomène de multiplication des écrans a bien été identifié et analysé par les médias et les théoriciens des média ${ }^{4}$, il nous parait important de rappeler que bien souvent la définition de l'écran n'est pas claire. Tantôt ce sont les contenus qui tiennent une part importante (l'écran de console de jeux pour diffuser des images de 
jeux vidéo, écran de télévision pour diffuser les signaux de télévision), tantôt ce sont les caractéristiques matérielles qui permettent de nommer toute « une nouvelle panoplie [issue] de la multiplication des terminaux " (Chantepie et Le Diberder 18). En effet, après le tube cathodique à l'œuvre dans «l'écran cathodique » (Roy 150), ce sont les cristaux liquides et le plasma qui seront à l'origine d'une nouvelle taxinomie de l'écran, puisqu'on peut depuis la fin du XX⿳亠丷⿵冂丶 ${ }^{\text {me }}$ siècle faire une distinction non plus en se basant sur le contexte d'utilisation, collectif ou individuel, privé ou public, mais sur la technologie déployée. Ainsi on pourra qualifier un dispositif d' "écran à cristaux liquides » (Roy 150) pour le différencier d'un « écran plasma » (Roy 151).

De plus, malgré une certaine permanence d'une sociologie des usages qui maintient la distinction entre les grands pôles de l'ère analogique (« on partage l'écran de la télévision alors qu'on monopolise celui d'un ordinateur » (Chantepie et Le Diberder 19), les déplacements entre intentions des fabricants et usages des publics ont contribué à brouiller les frontières qui séparaient certains écrans. D'abord récepteur du signal de télévision, le téléviseur est ensuite détourné pour diffuser d'autres images, celles des cassettes vidéo et celles des jeux vidéo (lancement de la console Odyssey de Magnavox et du magnétoscope VCR de Philips en 1972). On observe le même phénomène quelques années plus tard qui sera poussé à son paroxysme avec l'écran d'ordinateur qui développe ses fonctions dans une «logique de media center au cœur du réseau privé numérique connectant tous les appareils du foyer » (Chantepie et Le Diberder 19).

En appui à cette stratégie de mise en réseau, le développement d'Internet, "en permettant l'acheminement de données numérisées [et en] reliant entre eux des ordinateurs ou des réseaux locaux» («Internet»), contribue à renforcer ce principe d'indifférenciation des écrans reliés.

40 Cette figure du moniteur, du terminal ou du périphérique s'offre donc comme l'aboutissement de cette recherche de polyvalence de l'écran, mais rend problématique un projet de taxinomie.

\section{Proxémie}

41 Les mutations dans le domaine de l'image animée ont rendue obsolètes les critères de distinction entre les écrans utilisés depuis l'invention du cinématographe. L'importance de nouveaux concepts tels que l'interopérabilité - compatibilité technique des écrans entre eux et avec le système global de circulation des données - et la mobilité nous enjoignent à les prendre en compte pour élaborer une taxinomie adaptée aux mutations du secteur. Nous nous proposons donc de faire appel à la proxémie qui prend en charge à la fois l'aspect culturel et spatial de phénomènes de société.

Les termes proxémie et proxémique ont été introduits en France dans les années 1970, notamment par Abraham Moles et Elisabeth Rohmer. Ces chercheurs ont participé au développement du champ des sciences de l'information et de la communication, et en particulier à l'élaboration d'une psychologie de l'espace.

La première définition française de la proxémie confirme son ancrage transdisciplinaire, à la fois anthropologique et social, puisqu'elle fait référence à des "sciences qui traitent des problèmes du 'territoire de chacun' » («Proxémie »). Dans les années 1980, cette définition s'étoffe et la proxémie devient une «discipline 
scientifique qui étudie l'organisation signifiante de l'espace des différentes espèces animales et notamment de l'espèce humaine » ("Proxémie »). Algirdas Julien Greimas et Joseph Courtés proposent également à cette époque une définition de la proxémique appliquée à la sémiotique :

La proxémique est une discipline - ou plutôt un projet de discipline - sémiotique, qui vise à analyser les dispositions des sujets et des objets dans l'espace, et, plus particulièrement, l'usage que les sujets font de l'espace aux fins de signification. [...] Dans une première approche, la proxémique semble s'intéresser aux relations spatiales [...] qu'entretiennent les sujets entre eux, et aux significations non verbalisées qu'ils en retirent (Greimas et Courtés).

Adapté de l'anglo-américain proxemics, il s'agit à l'origine d'un terme proposé en 1963 par Edward Twitchell Hall. Cet anthropologue américain s'est spécialisé dans l'étude de la communication non verbale et de la perception culturelle de l'espace.

E. T. Hall s'est appuyé sur les différents modèles d'organisation spatiale rencontrés lors de ses voyages et sur les situations auxquelles il a été confronté au cours de ses missions professionnelles dans différentes régions du globe pour élaborer la définition de la proxémie :

Le terme de 'proxémie' est un néologisme que j'ai créé pour désigner l'ensemble des observations et théories concernant l'usage que l'homme fait de l'espace en tant que produit culturel spécifique (Hall 13).

En se basant sur l'observation du rapport de chacun à l'espace, il y distingue plusieurs dimensions, soit la distance physique qui sépare les individus les uns des autres, ou plus généralement les règles de l'organisation spatiale des dispositifs culturels d'une part, et la dimension subjective qui entoure chacun des éléments d'un dispositif d'autre part. Cette dimension subjective, E. T. Hall la qualifie de distance sociale :

La distance sociale n'est pas fixée avec rigidité, mais elle est en partie déterminée par la situation [...] Le téléphone, la télévision et les émetteurs portatifs ont allongé la distance sociale chez l'homme, permettant d'intégrer les activités de groupe très éloignées. L'extension de la distance sociale transforme aujourd'hui la structure des institutions sociales et politiques selon des modalités que l'on commence seulement à étudier. (Hall 29).

Le recours à la théorie de la proxémie de E. T. Hall nous semble pertinent dans le cas d'une étude portant sur l'écran vu l'importance qu'ont pris les aspects spatiaux et sociaux dans la conception et l'usage des écrans. En croisant des données sociales, anthropologiques, spatiales et ergonomiques, nous pensons qu'il est possible d'envisager une approche du dispositif écranique qui ne se fonde plus sur l'écran de projection mais qui prend en compte d'autres aspects révélés par les artefacts les plus récents.

\section{Nouvelle taxinomie}

Si les aspects collectif et public peuvent toujours être sollicités pour une taxinomie de l'écran, ils ont perdu de leur pertinence. Nous avons vu par exemple dans notre historique des écrans que ce qui pose problème avec certains artefacts, c'est qu'ils subissent une mutation, un « détournement » de leur fonction première. Dans le cas de l'écran de télévision, puis de l'écran d'ordinateur et enfin de l'écran du téléphone mobile, ces supports ont perdu leur spécificité pour devenir polyvalents. 
apparaît que d'autres critères intégrant les mutations sociales et technologiques des dernières décennies pourraient être plus appropriés pour établir une nouvelle taxinomie ou cartographie des écrans.

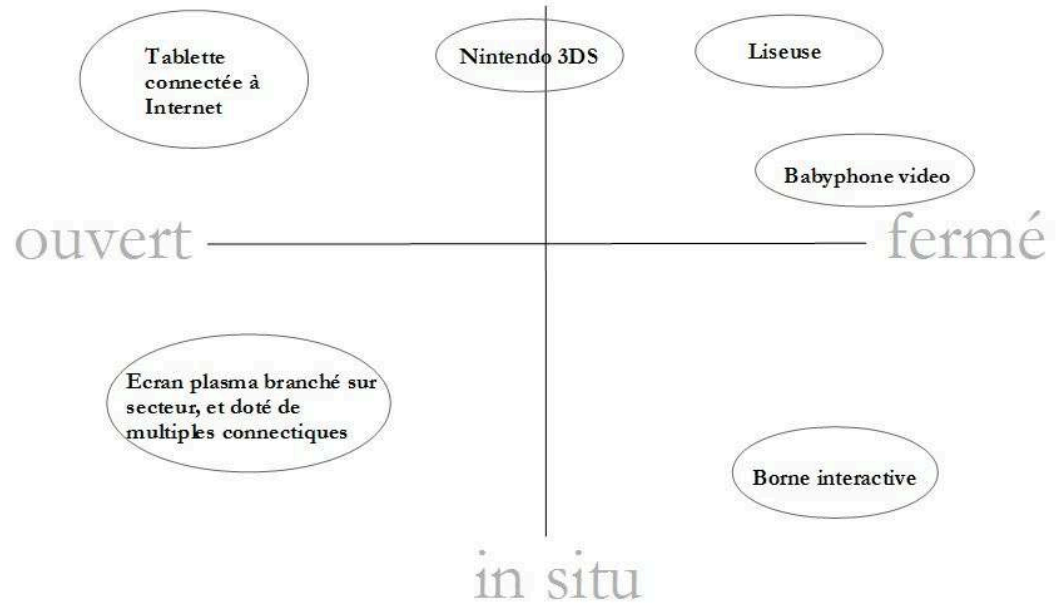

À la mobilité de certains dispositifs, nous avons choisi d'opposer le caractère in-situ d'autres écrans. Par in-situ, nous entendons le fait d'être situé de manière fixe dans l'espace, que l'on ait affaire à un élément architectural, tels les écrans intégrés aux façades de bâtiments, voire les façades de bâtiments elles-mêmes, ou qu'il s'agisse d'un dispositif scellé, localisable, modèle d' "organisation fixe» (Hall 132), installé de manière pérenne dans l'espace, comme la borne interactive.

51 Cet «appareil équipé d'un écran vidéo [qui] permet de délivrer à un utilisateur des informations sur un ou plusieurs sujets donnés » («Borne »), tire sa définition de celle

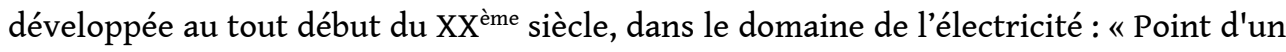
circuit électrique destiné à établir une connexion. Chacun des deux composants est destiné à raccorder un dispositif à des conducteurs extérieurs» («Borne»). Les archétypes du dispositif de la borne interactive sont par exemple la borne d'arcade dans le domaine du jeu vidéo, la borne de retrait dans le domaine du service (transactions bancaires, titres de transport) ou les bornes d'information (localisation sur un plan, médiation culturelle). Par opposition à ces écrans fixes, nous trouvons donc des écrans mobiles qui ont comme spécificité de n'être pas localisables par le biais de coordonnées géographiques fixes.

52 Le second axe oppose quant à lui les termes ouvert et fermé. Nous faisons référence ici au «mythe de l'interactivité » qui se décline selon Lev Manovich en deux modes d'interactivité, « ouverte et fermée » (Manovich 141), sujet également traité par Jeremy Rifkin dans sa description des enjeux de ce qu'il nomme "l'âge de l'accès »:

Le rôle de la propriété est en train de subir une transformation radicale. [...] Dans la nouvelle économie en réseau, plutôt que d'échanger des biens matériels et immatériels, les entreprises en contrôlent et en régulent l'accès. [...] La notion 
d'accès est un puissant outil conceptuel qui nous permet de repenser notre vision du monde et de l'économie (Rifkin 11-25). ces réseaux et de communiquer sur leur fonctionnement. La localisation des caméras et des zones d'émissions des images les rend tangibles et permet d'initier une prise de conscience quant à la structure des réseaux qui nous entourent. Ces projets peuvent se poursuivre par un second volet, lorsque des artistes utilisent les images captées pour réaliser des films, comme ce fut le cas pour Faceless (2007) de Manu Luksch ou The Duellists (2007) de MediaShed?

58 Ce qui nous intéresse ici, c'est le dispositif de visualisation et de captation des flux d'images sur lequel reposent certaines de ces performances. Pour $2.4 \mathrm{Ghz}$ de Recyclism 
(projet en cours depuis 2008) par exemple, les participants au projet de cartographie sont équipés de terminaux de réception d'images pour la vidéosurveillance domestique commercialisés sous le nom de babyphone video.

L'écran du babyphone video est un écran fermé, dédié à la diffusion d'images destinées aux proches de l'enfant, et ce dans un espace privé. Il est donc ici détourné puisqu'il est utilisé dans l'espace public, pour capter les flux d'images de caméras de vidéosurveillance installées par les particuliers, les municipalités ou les commerçants, ces derniers omettant de signaler la zone couverte :

The camera is intended to send its video signal to a nearby base station so that it can be viewed on a computer or a television. But its signal can be intercepted from more than a quarter mile away by off-the-shelf electronic equipment (video sniffing). (O'Rourke 300).

L'écran du babyphone video permet alors de détecter la présence des caméras puis de les localiser à partir de l'angle de prise de vue des images qui s'affichent. Dans le cas présent on a affaire à une forme de militantisme de la part des artistes qui ouvrent l'écran du babyphone video, mais certaines "effractions» se font aussi avec le consentement du fabricant.

L'exemple des écrans de la console de jeu portative Nintendo 3DS pour afficher des données, images et texte, en appui à une visite au musée du Louvre, relève en effet d'une forme de ré-emploi et non d'un détournement au caractère militant puisque le projet a reçu le soutien de Nintendo. Depuis 2012, dans le cadre d'un partenariat, le dispositif de la Nintendo 3DS, initialement dédié au jeu, a remplacé les audioguides au sein du musée du Louvre. Le changement de destination de l'écran de la console de jeu est signalé par l'emploi d'une terminologie spécifique, cette "nouvelle offre d'audioguidage » étant qualifiée « d'exceptionnelle » ( Louvre »).

Le caractère ouvert des écrans est comme on peut le voir un argument marketing et, de fait, il est généralement apprécié, voire plébiscité par les usagers. Le développement de l'écran de la tablette se fera en partie pour ces mêmes raisons au détriment de l'écran de la liseuse, qui bien qu'il puisse s'enorgueillir d'une technologie de pointe (papier électronique) qui procure un confort de lecture sans comparaison avec l'écran rétroéclairé de la tablette, n'a pas connu l'essor de cette dernière. La spécialisation de l'écran de la liseuse en fait un dispositif fermé ou perçu comme tel, alors que la polyvalence de l'écran de la tablette lui confère le statut de dispositif ouvert.

63 Audioguide, babyphone video, tablette, liseuse, ces écrans illustrent les changements induits par le «bouillonnement actuel des techniques [auquel] répond le temps long des usages et des lois de la création artistique " (Chantepie et Le Diberder 20). Mais, dans cette nouvelle cartographie de l'écran, quelle est donc la place de l'écran de cinéma?

S'il parait évident de le qualifier d'in-situ (pour l'instant), concernant le caractère ouvert ou fermé la réponse est moins claire. Pour répondre à cette interrogation, nous convoquerons le concept de "hors film», défini par la loi du 30 septembre 2010, relative à l'équipement numérique des établissements de spectacles cinématographiques :

L'installation d'équipements de projection numérique a permis le développement, au sein des salles de spectacles cinématographiques, d'une offre nouvelle (communément dénommée " hors film »), consistant en la diffusion de contenus dits « complémentaires » ou « alternatifs ». Il s'agit principalement de la diffusion, 
en direct ou différé, de spectacles vivants (opéra, ballets, concerts...) et

d'évènements sportifs ou culturels. ( $\mathrm{CNCa} 30$ ).

l'écran se manifestait surtout par le fait que le cinématographe permettait d'offrir le spectacle d'images en mouvement dans tous les lieux susceptibles d'accueillir un public suffisamment nombreux pour que la séance soit rentable. La pratique du cinéma itinérant, au sens où une projection nécessitait une installation pour une durée variable, dans les foires, les théâtres et music-halls, s'est progressivement raréfiée au profit de la projection dans des salles transformées ou construites à cet effet. Le potentiel d'adaptation de l'écran de projection s'est alors exprimé sous d'autres modalités, le dispositif variant en fonction des différents formats de films (taille, forme, appareillage variés ${ }^{9}$ ). À la lumière de ces éléments historiques, l'ouverture de l'écran de projection à d'autres images que celles des films de cinéma peut donc se concevoir comme une nouvelle étape dans l'histoire de cet objet polyvalent par nature.

69

Cette approche par le dispositif écranique pourrait par ailleurs être de toute utilité dans le cas de cet objet d'étude que représente l'expanded cinema. Les «querelles » qui ont lieu autour de ce "cinéma élargi ", "cinéma étendu», ou encore "cinéma contemporain» (Bellour 26-35) pourraient être éclairées d'un jour nouveau si l'on opposait au discours sur la question de la définition du cinéma les arguments en faveur d'une redéfinition de l'écran de cinéma à partir d'une taxinomie de l'écran renouvelée, en adéquation avec les mutations qui affectent la circulation des images et des spectateurs. 


\section{BIBLIOGRAPHIE}

Aumont, Jacques et Marie, Michel. Dictionnaire théorique et critique du cinéma. Paris : Armand Colin Cinéma, 2005.

Bellour, Raymond. La Querelle des dispositifs, Cinéma - installations, expositions. P.O.L. Trafic, 2012. Chantepie, Philippe et Le Diberder, Alain. Révolution numérique et industries culturelles. Paris : La Découverte, 2005.

Centre national du cinéma et de l'image animée (CNCa). Rapport sur la mise en œuvre de la loi No. 2010-1149 du 30 septembre 2010 relative à l'équipement numérique des établissements de spectacles cinématographiques. Mars 2012.

< http://www.cnc.fr/web/fr/rapports/-/ressources/ 1492550;jsessionid=9D4DADADB4DDAC1462690A5CB0EEFFE6.liferay >.

Centre national du cinéma et de l'image animée (CNCb). Les principaux chiffres du cinéma en 2014. Mars 2015.

<http://www.cnc.fr/web/fr/detail_ressource?

p_p_auth=2rOQH85L\&p_p_id=20\&p_p_lifecycle $=1 \&$ p_p_state $=$ exclusive\&p_p_mode $=$ view\&p_p_col_id $=$ column- $1 \&$ p_p_col_count $=1 \&$ str $>$.

Centre national du cinéma et de l'image animée (CNCc). Les chiffres clés du cinéma français en 2014 dévoilés avant Cannes. Paris, le 07 mai 2015.

< http://www.cnc.fr/web/fr/actualites/-/liste/18/6949146 >.

De la Cotardière, Philippe (dir.) Histoire des sciences de l'Antiquité à nos jours. Paris : Taillandier, 2004.

Désile, Patrick. Généalogie de la lumière, du panorama au cinéma. Paris : L'Harmattan, 2000.

Francoeur, Louis Benjamin et al. Dictionnaire technologique ou nouveau dictionnaire universel des arts et métiers et de l'économie industrielle et commerciale. Tome 4. Paris : Thomine, 1822-1835.

Fourmentraux, Jean-Paul. « Net art. ». Communications, 88, sous la direction de Antonio A. Casilli. Seuil, Cultures du numérique, (2011) : 113-120.

Furetière, Antoine. Dictionnaire universel contenant généralement tous les mots français tant vieux que modernes et les termes de toutes les sciences et des arts. La Haye/Rotterdam : Arnout \& Reinier Leers, 1690.

Greimas, Algirdas-Julien et Courtés, Joseph. Sémiotique : dictionnaire raisonné de la théorie du langage. Paris : Hachette supérieur, Hachette université, 1993.

Hall, E. T. La dimension cachée. Paris : Seuil, Points Essais, 1971.

Icher Bruno et Péron Didier « Le hors-film entre en salles », in Libération, 16 juin 2010. 4 Septembre 2013.

< http://www.ecrans.fr/Le-hors-film-entre-en-salles,10124.html >.

Jullier, Laurent, L'écran post-moderne. Un cinéma de l'allusion et du feu d'artifice. Paris : L'Harmattan, 1997.

Lemoine, Stéphanie et Ouardi, Samira. Artivisme: Art, action politique et résistance culturelle. Paris : Alternatives, Arts urbains, 2010. 
« Louvre. » Site internet du musée du Louvre. 15 Août 2013.

< http://www.louvre.fr/l-audioguide-du-musee/pratique\#tabs >.

Luksch, Manu et Patel Mukul (dir), Ambient Information Systems. Londres : AIS, 2009.

Mongin, Olivier et Padis, Marc-Olivier. « La société des écrans et la télévision ». Esprit (mars 2003) : 8-12.

O'Rourke, Karen. Walking and Mapping: Artists as Cartographers. Cambridge : The MIT Press, 2013.

Manovich, Lev. Le langage des nouveaux médias. Paris : Les presses du réel, 2010.

Rifkin, Jeremy. L'âge de l'accès. La nouvelle culture du capitalisme. Paris : La découverte, 2005.

Roy, André. Dictionnaire général du cinéma. Du cinématographe à internet. Québec : FIDES, 2007.

Sicard, Monique. L'année 1895, l'image écartelée entre voir et savoir. Paris : Synthélabo, Les

empêcheurs de penser en rond, 1994.

Stocker Gerfried et Christine Schöpf (dir). Ars Electronica 2007: Goodbye Privacy-Welcome to the Brave New World. Ostfildern : Hatj Ceantz, 2007.

\section{Dictionnaires et Encyclopédies en ligne}

"Borne." 15 Août 2013.

< http://www.larousse.fr/dictionnaires/francais/borne/10264/locution >.

"Écran." Centre National de Ressources Textuelles et Linguistiques. 15 Août 2013.

< http://www.cnrtl.fr/definition/dmf/écran >.

"Oscilloscope cathodique." Encyclopédie Universalis. 15 Août 2013.

< http://www.universalis.fr/encyclopedie/oscilloscope-cathodique/ >.

"Proxémie." Centre National de Ressources Textuelles et Linguistiques. 15 Août 2013.

< http://www.cnrtl.fr/lexicographie/proxémie >.

"Scherm." Centre National de Ressources Textuelles et Linguistiques. 15 Août 2013.

$<$ http://www.cnrtl.fr/definition/dmf/écran?idf=xxx;etym=scherm >.

\section{NOTES}

1. La date du 28 décembre 1895, au Salon Indien du Grand Café à Paris, a été retenue comme événement historique car il s'agissait de «la première projection publique payante du cinématographe des frères Lumière » (Sicard 7). Ce sont donc ces deux aspects, collectif et public, qui la différenciaient des projections antérieures.

2. On peut citer notamment les revues La Scène et l'écran. Revue bi-mensuelle du théâtre et du cinématographe (1914), L'Écran parisien. Organe pratique des spectacles cinématographiques (1917), L'Écran d'outre-Méditerranée. Le premier journal cinématographique de l'Afrique du Nord (1927) auxquelles s'ajoutent des ouvrages, tels que Vedettes mondiales de l'écran de Gabriel S. Patlagean (1923), L'idée et l'écran : opinions sur le cinéma d'Henri Fescourt et Jean-Louis Bouquet (1925).

3. Dans les années 2000, on a vu apparaître l'expression «société des écrans " (Mongin et Padis) qui faisait référence à la surabondance d'images, due à l'accroissement du nombre de chaînes de télévision et aux débuts de la diffusion d'images sur de nouveaux supports comme l'écran d'ordinateur. En quelques années, de nombreuses autres locutions utilisant le terme écran au 
pluriel ou au singulier ont vu le jour pour désigner un phénomène complexe et global touchant à la fois l'augmentation de l'offre de contenus, la diversification des dispositifs écraniques (commercialisation des pagers et des assistants numériques personnels, puis des téléphones et des tablettes) et la généralisation de l'usage des écrans aussi bien dans l'espace public (à des fins de publicité, de signalisation ou d'information) que privé (développement de la domotique).

4. Voir notamment des travaux tels que ceux réalisés lors du programme «Écrans » durant la période 2008-2012 (Université de Genève, Université de Manchester, Université de Marrakech, Université Michel de Montaigne, Bordeaux 3), la revue Écranosphère (dirigée par Julie Beaulieu, Université de Laval) ou encore la revue Écrans (éditée par L"Harmattan).

5. Proposé par Hans Robert Jauss à partir de Gadamer et Heidegger, il s'agit d'un concept utile aux études sur la réception, pour mesurer l'écart existant entre une œuvre et l'attente du public (conditionnée par deux aspects de son expérience : celle qu'il a de la vie en général et celle qu'il a du genre dont l'œuvre relève).

6. Dans le sens donné par l'Open Source Initiative de Palo Alto, c'est à dire de technologies qui offrent des possibilités de libre redistribution, un accès au code source et encouragent la création de travaux dérivés.

7. L'emploi des images captées par les systèmes de vidéosurveillance fait l'objet d'une véritable réflexion de la part des artistes vidéastes, qui a abouti à la rédaction du « Manifesto for CCTV Filmmakers » (Luksch et Mukul 271-274) en 2004.

8. « En 2014, [...] 0,5\% des entrées sont réalisées par le hors flm. » (CNCb)

« En 2014, [...] le hors film génère plus de 1,05 million d'entrées (+77,9 \%). » (CNCC)

9. Voir notamment l'analyse que fait Laurent Jullier des innovations telles que l'I-max (Jullier 85), le Cinémascope (Jullier 53), l' « effet de bain » (Jullier 60) ou encore le «Cinéma kinésique » (Jullier 85)

\section{RÉSUMÉS}

Apparue à la fin du XIX ème siècle, la projection cinématographique se fonde sur un dispositif constitué de deux objets caractéristiques que sont le projecteur et l'écran. Or, si la conception du projecteur est bien contemporaine de l'art auquel il a donné naissance, il faut rappeler que l'histoire de l'écran commence en amont de celle du $7^{\text {ème }}$ art.

L'écran de cinéma tendant à éclipser les modèles qui le précédaient, nous reviendrons sur ces formes écraniques, envisageant l'apparition du terme écran et l'évolution de sa définition comme autant d'indices d'un phénomène culturel en devenir.

Ces éléments historiques seront un préalable pour mener une étude sur des dispositifs contemporains qui questionnent les limites du champ d'action de leurs concepteurs sur les pratiques des usagers.

En mettant en perspective ces différents artefacts à l'aide d'une approche proxémique, nous nous attacherons à démontrer leur filiation, témoignage d'une histoire de l'écran qui, bien qu'elle croise celle du cinéma, s'en distingue néanmoins.

Created at the end of the $19^{\text {th }}$ century, the cinematograph distinguished itself from other moving pictures apparatus since it combined both a projector and a screen. Although the projector was specifically designed for projecting movies, the screen already had a long history of its own.

This is a fact that tends to be forgotten, as it is quite notable that the cinema screen has 
overshadowed all previous forms of screen. A study of these different models will contribute to the analysis of contemporary devices which border the field of the cinema.

After a brief study of the history of the screen, it will be interesting to consider its evolution as an indicator of a cultural phenomenon in connection with the movie industry. Finally, we will list and map out some interesting post-cinematographic screens to show how the scope of their use, set out by their designers, can limit the practices of their users. Proxemics, as a science whose purpose is to study the spatial organization of cultural features, will help shed light on the links between these various artefacts throughout the history of the cinema.

\section{INDEX}

Keywords : screen, screens, proxemics, circulation, interaction, mapping, experience, cinema, device, apparatus, taxonomy, interoperability, mobility

Mots-clés : écran, écrans, proxémie, circulation, interaction, cartographie, expérience, cinéma, dispositif, taxinomie, interopérabilité, mobilité

\section{AUTEURS}

\section{CÉCILE MARTIN}

Ingénieur conseil média

Université Paris 1 Panthéon Sorbonne 